\title{
Current Applications and Future Promises of Machine Learning in Diffusion MRI
}

\author{
Daniele Ravi ${ }^{[0000-0003-0372-2677]}$, Nooshin Ghavami ${ }^{[0000-0002-4310-1245]}$, \\ Daniel C. Alexander ${ }^{[0000-0003-2439-350 X]}$, and Andrada \\ Ianus $^{[0000-0001-9893-1724]}$ \\ University College London d.ravi@ucl.ac.uk
}

\begin{abstract}
Diffusion-Weighted Magnetic Resonance Imaging (DW-MRI) explores the random motion of diffusing water molecules in biological tissue and can provide information on the tissue structure at a microscopic scale. DW-MRI is used in many applications both in the brain and other parts of the body such as the breast and prostate, and novel computational methods are at the core of advancements in DW-MRI, both in terms of research and its clinical translation. This article reviews the ways in which machine learning and deep learning is currently applied in DW-MRI. We will also discuss the more traditional methods used for processing diffusion MRI and the potential of deep learning in augmenting these existing methods in the future.
\end{abstract}

Keywords: Diffusion-Weighted · MRI · Machine Learning · Deep Learning

\section{Introduction to diffusion MRI}

DW-MRI probes the random motion of diffusing water molecules in biological tissue [1]. Since the diffusion pattern is influenced by the presence of cellular membranes and their configuration, DW-MRI is an indirect probe of tissue structure at the microscopic scale [2]. Standard DW-MRI measurements are made by applying a pair of magnetic field gradients with opposite polarities, usually referred to as diffusion gradients, which encode displacement along their direction [1].

In DW-MRI contrast, the larger the molecular displacement, the more the signal is attenuated. Thus, tissues with highly packed cells, such as some tumours, appear bright, and areas where water diffuses freely, such as cerebral spinal fluid, appear dark [3,4]. DW measurements are often described in terms of their gradient orientation and b-value, which reflects the diffusion weighting power and depends on the gradient strength, duration and diffusion time [2].

The simplest DW-MRI technique is mapping the Apparent Diffusion Coefficient (ADC), which requires at least two measurements, with and without diffusion gradients. The contrast in an ADC map is inverted from the DW-MRI contrast. Thus areas of low ADC values correspond to tissue with tightly packed cells, while high ADC values correspond to areas where molecules can diffuse 
more. The simple ADC model assumes isotropic diffusion, thus it is often used in stroke and cancer imaging [3,4].

In order to probe neural tissue, which is highly anisotropic in white matter, DW-MRI techniques need to account for anisotropic diffusion. Thus, a methodology commonly applied for brain imaging is Diffusion Tensor Imaging (DTI) [5], which is an extension of the diffusion coefficient in three dimensions, and requires at least $6 \mathrm{DW}$ measurements with non-colinear gradient directions to be estimated. DTI can report on the main fibre orientation and provides scalar metrics which reflect diffusion properties of the tissue, for instance, axial and radial diffusivity or fractional anisotropy. DTI is employed in a wide range of applications involving brain [6] and central nervous system [7] imaging. Such applications include mapping white matter tracts [8], studying development [9] and ageing [10] as well as investigating various diseases, such as, Multiple Sclerosis (MS), Alzheimer's disease, diffuse axonal injury, etc $[11,12]$. Although DTI is simple and robust, it cannot describe multiple fibre populations and/or diffusion in a more complex environment with restricting barriers [13].

Over the last decades, a significant effort has been made to improve the measurement protocols and data analysis tools for DW-MRI in order to have better sensitivity and specificity to various tissue characteristics, while keeping the acquisition time clinically feasible [14-16].

Novel computational methods, alongside improvements in scanner capabilities, are at the core of advancements in DW-MRI, both in terms of research and its clinical translation. The rest of the paper will look at the different methods currently used for acquiring, processing and applying DW-MRI, with a focus on machine learning which is becoming an important tool used to efficiently solve computational problems in this wide range of applications.

\section{Mathematical vs Computational Modelling}

Current research on DW-MRI includes the development of methods for the entire imaging pipeline, for instance designing better acquisitions [17], image reconstruction [18], microstructure parameter mapping [19, 20], super-resolution [21], tractography [22], parcellation [23], detection of lesions [24], monitoring treatment response [25], etc. State-of-the-art methods on DW-MRI can be divided into two main categories: those based on mathematical modelling and the others based on machine learning, as shown in Figure 1. Usually, mathematical modelling exploits a set of assumptions or theoretical foundations $[26,27]$ to model some physical property of the DW-MRI. Instead, the methods in the latter category are data-driven computational methods that model the output from its input while making the smallest number of assumptions possible.

In general, mathematical models are used when the process that needs to be described is partly or completely known (e.g. in the case of image reconstruction or mapping parameters in DTI). However, sometimes accurate mathematical models that express relationships to the DW-MRI signal are intractable. In such situations, as well as when a more complex and largely unknown phenomenon 


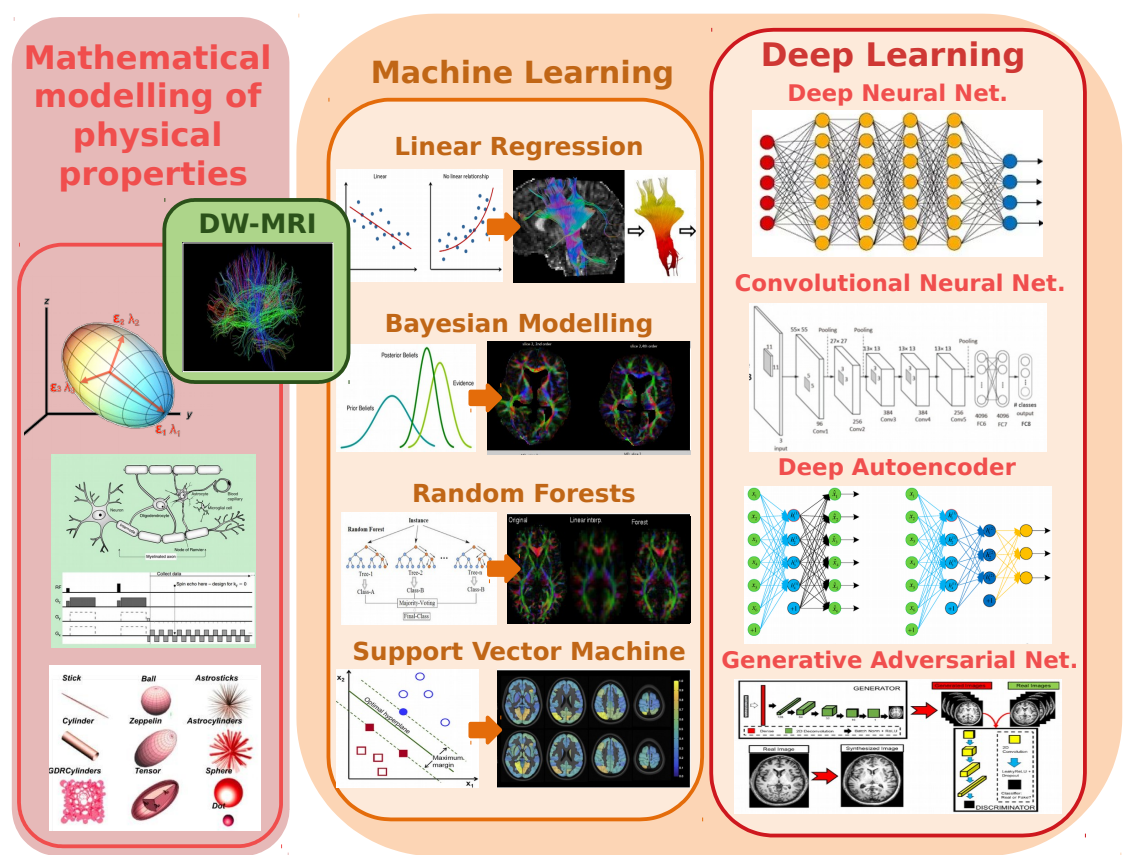

Fig. 1. Main models used in the processing and analysis of DW-MRI.

is under examination, computational methods and especially machine learning methods are more adequate. In particular, in the last few years, a sub-category of machine learning called deep learning has become very popular [28].

The theoretical principles behind deep learning are based on the Artificial Neural Network (ANN) concepts. The main difference between the basic ANN and advanced deep learning is that the latter exploits more hidden neurons, more layers and new training paradigms to process a larger amount of data. This results in an effective high-level abstraction enabling automatic feature extraction, which otherwise would have to be done by explicitly deriving a set of hand-crafted features before applying the model.

Rapid improvements in computational power, fast data storage, and parallelisation have contributed to making deep learning very popular. This also applies to the field of DW-MRI and is confirmed from our analysis shown in Figure 2. In Figure 2 a) we can see that the number of papers published today exploiting machine learning or deep learning methods is comparable to the number of papers based on mathematical modelling, and, as explicitly illustrated in Figure $2 \mathrm{~b}$ ), since 2010 their numbers increased much faster compared to the papers based on mathematical approaches which instead have remained approximately unaltered. 


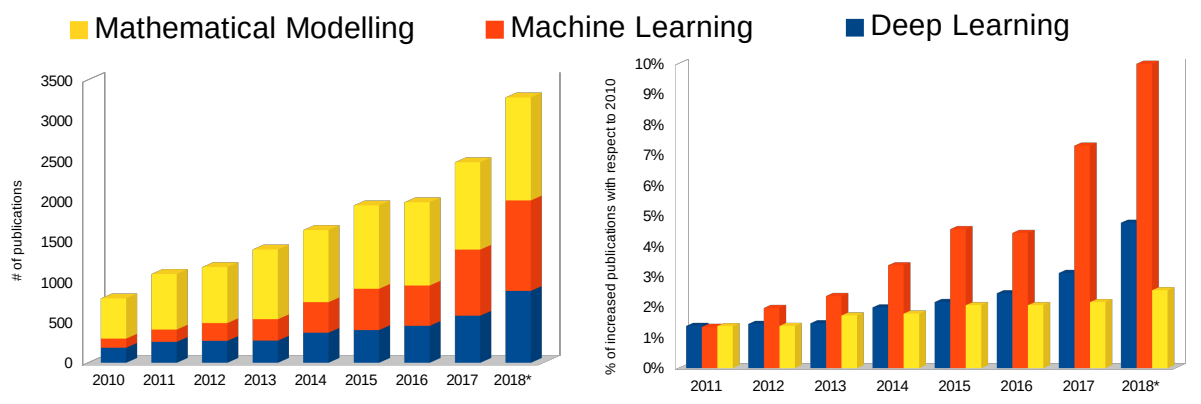

Fig. 2. a) Distribution of published papers in the field of DW-MRI. b) Percentage of increased publications with respect to 2010. The results are obtained from Google Scholar; the search phrase is defined using the key word DW-MRI plus the name of popular models in the correlated category e.g., "DW-MRI" "Deep learning" OR CNN OR Autoencoder.

For this reason, in this chapter, we focus our analysis mainly on methods based on machine and deep learning, (i.e. the orange section of Figure 1) providing a comprehensive up-to-date overview of the current state-of-the-art in DW-MRI. Both the machine learning and deep learning parts will be subdivided into different methods, within which a variety of applications will be described, as summarised in Table 1.

\section{Standard Machine Learning Models}

In this section, we focus our analysis on the main machine learning methods used for processing DW-MRI. We will divide the section up into the different methods and describe the applications that each is used for.

\subsection{Linear Regression}

Linear regression is the simplest machine learning approach, which linearly models the response of a dependent variable to one or more independent variables. It can be subdivided into simple linear regression (when there is only one independent variable), or multiple linear regression (which as the name suggests involves more than one independent variable). In the following paragraphs, we will describe some of the applications in DW-MRI.

Linear regression is mainly used to fit many of the standard models to DWMR data such as ADC, DTI, etc. [29] and it has shown to be particularly useful to study changes in diffusion metrics, for instance with disease or ageing. One application of this method is for distinguishing benign and malignant tumours in the breast as described in [24]. Linear regression was used in this last paper to compare mean $\mathrm{ADC}$ values and cellularity values between the different types 
of tumours and was able to show statistically significant differences between the malignant and benign tumours for both measures.

Another application of this method was demonstrated in [30] for rectal cancer. The authors use the fact that ADC values of water in a tumour can show how much necrosis there is, to find a correlation between these ADC values and responses of a tumour to chemotherapy treatment. Using the regression model, the authors report a negative correlation between $\mathrm{ADC}$ values before and after chemotherapy. Finally, the authors in [31] focus on the age-related microstructural differences through DTI. By computing diffusion parameter maps and using a mass-univariate linear regression model on these maps as part of the analysis, fractional anisotropy in white matter area was shown to decrease with age in a linear fashion.

These class of models do come with a few limitations. Firstly, as the name suggests, they are limited to only modelling linear relationships, and this isn't necessarily the case with many data. In addition to this, the method is also limited by the fact that it can be very sensitive to outliers, and these outliers can significantly affect the regression results.

\subsection{Random Forest}

Random Forests (RF) are an ensemble learning method and a type of supervised learning algorithm which can be used for both classification and regression tasks. It works by constructing a number of decision trees (forest) during training, one to each bootstrap sample drawn from the full set of training data. Once trained, the model typically outputs the 'majority' class in classification tasks or the 'mean' class for regression over the full set of trees in the forest. RF has been used in a wide range of applications, such as super-resolution, correlation of biomarkers with disease/ageing, classification of lesions, microstructure parameter mapping etc.

Initially, RF has been employed to tackle the intractability for some of the mathematical models of DW-MRI, such as in the microstructural parameter mapping task. For example, Nedjati-Gilani first in [32] and later in [20], proposed an RF approach to map permeability in the brain. In particular, they highlight that traditional mathematical models of diffusion through semi-permeable membranes assume strong mixing, i.e. the average diffusing particle spends time in multiple environments during the experiment. However, this assumption breaks down with typical brain-compartment sizes and diffusion times in DWI. The computational model encoded in the RF is able to learn an implicit model from Monte-Carlo simulations thereby providing a mechanism to solve the inverse problem of estimating tissue parameters from measurements. Computation times prevent usage of the simulation directly for this purpose. A similar approach is presented in [33] to map axon diameter from DW-MRI with a RF trained on matching histological data.

Other DW-MRI related applications of RF are for studying biomarker changes. For example, [34] assesses the correlation between cognitive impairment and structural damage in the central nervous system for MS. RF has been shown to 
be useful not only for diseased states but also in healthy ageing as can be seen in [35] which use a Conditional Inference RF on DTI to investigate reductions in white matter integrity with age.

In [36] a RF classifier has been combined with a voting procedure to directly processing raw diffusion-weighted signal intensities and obtain fibre tractography. Quantitative and qualitative evaluation show the potential of this technique in comparison with the tractography pipelines that rely on mathematical modelling. RF has been also used to solve classification problems, for example to segment lesion volumes on multiparametric-MRI (mp-MRI), including ADC maps, in patients following ischemic stroke, as detailed in Mitra et al. [37]. RF classifiers showed high accuracy for the detection of these lesion areas.

An emerging application enabled by the use of RF is the Image Quality Transfer (IQT) [21]. In this context, Alexander proposed a method to learn and transfer the fine structural detail of images in high-quality data sets, which have longer acquisition times, to enhance lower quality data sets, which have more standard acquisition times. Later in [38] the same authors used the proposed method on the Human Connectome Project (HCP) showing, in more details the benefits of this quality transfer for both brain connectivity mapping and microstructure imaging. However, the aforementioned approaches do not give any indication of confidence in the output, which can be a significant barrier to adopting IQT in clinical practice. For this reason, Tanno et al. [39], presented a Bayesian formulation of RF which enables efficient and accurate quantification of uncertainty in terms of enhanced super-resolution performance.

Even with the many applications of RF described, this machine learning model does have its shortcomings. The main limitation of RF is its poor generalisability outside the training set, due to overfitting, particularly when presented with noisy data.

\subsection{Bayesian Models}

Bayesian modelling, also known as Bayesian inference, relies on Bayes' theorem for updating the probability of a hypothesis as more evidence is obtained. Bayesian modelling is especially useful when data is limited, allows the problem of overfitting to be avoided and is also useful as an approach for modelling uncertainty [40,41]. For example, Reisert et al. [19] have proposed a supervised machine learning approach based on a Bayesian estimator to disentangle the microscopic cell properties of the human brain from the effects of the mesoscopic structure. Bayesian approaches also have an application in the field of tractography. The model presented in [42] is used to investigate the uncertainty associated with estimated white matter fibre paths, in addition to computing the probability of connections between different brain areas. The model is both simple to implement and has the ability to handle noise.

The main limitation when it comes to Bayesian approaches is the requirement of a prior, which can sometimes be very difficult to formulate. In terms of the computation power, this approach also requires a high computational cost, especially for more complex models with a large number of parameters. 
Table 1. Table containing references and applications that use machine learning and deep learning for DW-MRI.

\begin{tabular}{|c|c|c|c|c|}
\hline Class & Target & Method & Task & Ref. \\
\hline \multirow{16}{*}{ 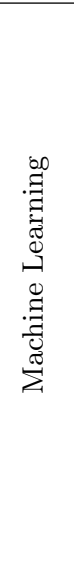 } & Breast & Linear regression & Lesions classification & {$[24]$} \\
\hline & Rectum & Linear regression & Malignant tumour classification & {$[30]$} \\
\hline & Brain & Linear regression & Age-related microstructural differences & {$[31]$} \\
\hline & Brain & RF & Permeability map & {$[32,20]$} \\
\hline & Brain & $\mathrm{RF}$ & Assess biomarkers correlation & {$[34]$} \\
\hline & Brain & $\mathrm{RF}$ & Investigate brain changes & {$[35]$} \\
\hline & Brain & $\mathrm{RF}$ & Tracrography & [36] \\
\hline & Brain & $\mathrm{RF}$ & Lesion classification & {$[37]$} \\
\hline & Brain & $\mathrm{RF}$ & Image quality transfer & {$[21,38]$} \\
\hline & Brain & Bayesian model & Estimate microstructural properties & [19] \\
\hline & Brain & Bayesian model & Investigate uncertainty in tractography & [42] \\
\hline & Brain & SVM & Survival time prediction & [43] \\
\hline & Brain & SVM & Classification of high angular resolution & [44] \\
\hline & Brain & SVM & Decoding gender dimorphism & [45] \\
\hline & Prostate & $\mathrm{CRF}$ & Cancer localization & [46] \\
\hline & Prostate & RVM & Cancer localization & {$[47]$} \\
\hline \multirow{20}{*}{ 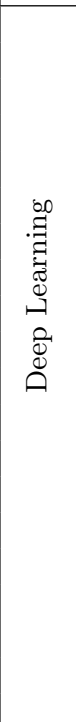 } & Brain & ANN & Predicting ischemic infa & {$[48]$} \\
\hline & Brain & ANN & Parameter mapping & [49] \\
\hline & Brain & DNN & Image acquisition & {$[50,51]$} \\
\hline & Brain & DNN & Permeability estimation & {$[52]$} \\
\hline & Brain & DNN & Survival time prediction & {$[53]$} \\
\hline & Brain & DNN & Cancer detection & {$[54]$} \\
\hline & Brain & $\mathrm{CNN}$ & Estimation of fiber orientations & {$[55]$} \\
\hline & Rectum & $\mathrm{CNN}$ & Cancer detection & [56] \\
\hline & Brain & $\mathrm{CNN}$ & White matter tract segmentation & {$[57]$} \\
\hline & Prostate & $\mathrm{CNN}$ & Cancer segmentation & [58] \\
\hline & Brain & $3 \mathrm{D}-\mathrm{CNN}$ & Survival time prediction & {$[59]$} \\
\hline & Kidney & U-net & Image registration & {$[60]$} \\
\hline & Brain & Bayesian CNN & Image quality transfer & [61] \\
\hline & Brain & $\mathrm{CNN}$ & Image quality transfer & {$[62]$} \\
\hline & Prostate & Deep autoencoder & Cancer grade groups & [63] \\
\hline & Brain & Deep autoencoder & Spatio-temporal denoising & {$[64]$} \\
\hline & Kidney & Deep autoencoder & Classification of renal rejection types & {$[65,66]$} \\
\hline & Brain & VAE & Detection of q-space abnormalities & {$[67]$} \\
\hline & Brain & GAN & Spatial super-resolution & [68] \\
\hline & Prostate & GAN & Semantic segmentation & [69] \\
\hline
\end{tabular}

\subsection{Support Vector Machines}

One of the most popular machine learning approaches is the class of Support Vector Machines (SVMs). SVMs are supervised learning models used for both classification and regression, based on the concept of finding the best set of hyperplanes in a high dimensional feature space that best separates the different classes. Intuitively, a good separation is achieved by the hyperplanes with the largest distance to the nearest training data point of any class. One of the main advantages of SVMs over the other methods described so far is its ability to 
generalise well and avoid overfitting. This is due to the fact that once the SVM has found the optimum hyperplane, any small changes to the data won't be able to greatly affect the hyperplane.

SVMs have been widely used in brain and cancer imaging for many applications, such as to estimate the survival rates in patients with grade II-IV gliomas from diffusion and perfusion-weighted MRI [43] or to classify High Angular Resolution Diffusion Imaging (HARDI) in vivo data [44]. In this last approach the SVM was trained to discriminate between six different classes: parallel neuronal fibre bundles in white matter, crossing neuronal fibre bundles in white matter, grey matter, partial volume between white and grey matter, background noise and cerebrospinal fluid. The features used were rotation invariant and obtained by exploiting a spherical harmonic decomposition of the HARDI signal.

Another interesting approach based on SVM was developed in [45], in order to perform multimodal classification and exploit the relative strengths of each modality. The paper is based on the idea that female brains contain a larger proportion of grey matter tissue, whereas the male brain consists of more white matter, and this can be used to study dimorphism of the human brain. However, the authors show that a single imaging modality is not enough and instead mp-MRI, through the use of T1, T2, and DW-MRI, is required to obtain good performance. The results from their study show that combining these different modalities yields a significantly higher balanced classification accuracy than using a single one. On the same wave is the approach developed in [46] for localizing cancer within the prostate, also through the use of mp-MRI that includes Dynamic Contrast Enhanced (DCE) MRI, DW-MRI and T2-weighted MRI. Here, the authors developed a new segmentation method that combines a Conditional Random Field (CRF) with a cost-sensitive framework. The CRF is a class of statistical modelling methods used in machine learning to include structural information in the prediction. For the similar task of prostate cancer localization, Ozer et al. [47] propose, instead, to combine the pharmacokinetic parameters derived from DCE MRI with T2 MRI and DW-MRI. To process these images, they use a Bayesian formulation of the SVM called Relevance Vector Machines (RVM). The accuracy obtained by the RVM has been compared with the SVM and results showed that it can produce more accurate and more efficient segmentation.

Although SVMs have been used in many applications, they suffer from an interpretability problem, with both the final model and the variable weights being hard to understand. SVMs, as well as the other 'classical' machine learning methods described so far, also suffer from a few general limitations. Firstly, an important issue is scalability. Even when these classical methods work well on small amounts of data, they fail to give the same accuracy on larger datasets and would require significantly larger computational power to analyse. Another big limitation is the need for complex feature engineering, a challenging task which requires a significant amount of time and effort for finding the best features. Moreover, these features are often limited in terms of adaptability or transferability to other domains/applications and extensive study within each different 
area may be required to transfer their usability. All of these major limitations can be overcome by the introduction of deep learning, which is the focus of the rest of the paper.

\section{Deep Learning}

In the last few years, requests of processing large amount and complexity of data, have driven the evolution of machine learning to the so-called deep learning paradigm. Although deep learning is a relatively new field, many different models have already been proposed. The design of a deep learning model mainly depends on: i) the amount of data available during the training and the related data-dimensionality (e.g. scalar data, images, 3D images), ii) the training procedure (supervised or unsupervised), iii) the desired goal (e.g. classification, regression, segmentation), and iv) last but not least, the computation resources (cloud computing, Graphics Processing Units (GPU), memory, Central Processing Units (CPU), etc.).

\subsection{Artificial Neural Network}

Before starting to describe the advanced methods of deep learning we will briefly overview the foundation behind it which comes from the ANN theory. An ANN is an ensemble of perceptrons, where a perceptron is defined by a non-linear transfer function $f$ and by two set of parameters: $W$ (the weights) and $b$ (the bias). The output of each neuron is the linear combination of the input $x$ with the $W$ added to the bias $b$, followed by the application of the transfer function (i.e. sigmoid or hyperbolic tangent function). One of the most popular ANNs is the Multi-Layered Perceptrons (MLP) that organizes the neurons in many different layers.

ANNs are usually trained through different steps, where at each step a new input sample or batch of samples are presented to the network. In this process, the weights are adjusted using a delta rule and a back-propagation function. Initially, random values are usually assigned to the network parameters and through this iterative training process, the parameters are updated to minimize the difference between the network predictions and the desired outputs.

In DW-MRI, ANN has been successfully used, for example, to determinate a voxel-by-voxel forecast of the chronic T2-Weighted images with the purpose of predicting ischemic infarction [48] or for a voxelwise parameter estimation of the combined intravoxel incoherent motion and kurtosis model in [49].

\subsection{Deep Neural Network}

To abstract more complex concepts or extract more sophisticated features, many hidden layers can be added to an ANN, defining a deep architecture referred to as a Deep Neural Network (DNN). Training a DNN is not always trivial, mainly due to possible numerical instabilities that could make the updated weights 
negligible (vanishing gradient problem), and adequate training countermeasures must be considered to avoid these issues.

DNN in DW-MRI was used in many applications from improving acquisition to parameter mapping and studying patient outcome. For example, [50] combined a DNN with spherical harmonics to learn a non-linear mapping that makes it possible to augment existing shells exploiting shells already acquired. This can be particularly useful to reduce the acquisition time of HARDI data. On a similar task, Golkov et al. [51] proposed a method called q-space deep learning which allows mapping scalar parameters, such as diffusion kurtosis or orientation dispersion from significantly reduced acquisitions and detecting abnormalities without the intermediate steps of diffusion models.

The authors in [54] proposed a Deep Learning Diffusion Fingerprinting (DLDF) method based on DNN and used to classify DW-MRI voxels. The authors showed that this model can learn even with limited training samples, and the method is capable to segment brain tumours, distinguish between young and older tumours and detect whether or not a tumour has been treated with chemotherapy. The paper concluded also that DLDF can localize changes in the underlying tumour microstructure, which are not evident using conventional measurements of the ADC. Mapping tissue microstructure features, especially permeability, is also investigated in [52], where the authors show that the results provided by the DNN are better than those obtained from a RF regressor model. DNN has also been used to post-process DW-MRI images and predict, for example, survival time in amyotrophic lateral sclerosis using as input data the structural connectivity and brain morphology [53].

\subsection{Convolutional Neural Network}

The main limitation of DNNs is that they do not scale well with multidimensional inputs, such as an image. The reason for this is that the number of nodes and the number of parameters required by a DNN in these cases could become very large, and therefore, their use is not practical. Convolutional Neural Networks (CNNs) have been proposed exactly to overcome these issues and today they are the most successful models for image analysis in deep learning. The design of a CNN is largely inspired by the neurobiological model of the visual cortex and can be summarized in three main steps: i) The input image is convolved using several small kernels, ii) the output of the previous step is subsampled and iii) the first 2 steps are repeated until high-level features are extracted. Differently from DNNs, CNNs use shared weights -the kernels- to perform the convolutions. This enables the network to process efficiently multidimensional data such as medical images. In the field of DW-MRI, CNNs are mainly used in the postanalysis step, for example, to perform tractography, for tissue segmentation or for predicting survival time.

In [55] a CNN is used to reconstruct fibre orientation, which is useful for studying brain connectivity. Rather than fitting a diffusion model, the authors proposed to solve a voxel classification problem in order to estimate the fibre orientation. In [56], instead, a CNN was trained for segmentation of tumours 
in patients with rectal cancer on mp-MRI, classifying each voxel into a tumour or non-tumour class. On a similar task, Clark et al. [58] proposed a CNN to delineate the prostate gland. The proposed algorithm first detects the slices that contain a portion of the prostate gland within the three-dimensional DW-MRI volume and then uses this information to segment the prostate gland and the related transition zone.

TractSeg proposed by Wasserthal et al. in [57] is also a method based on CNNs for fast and accurate white matter tract segmentation. This novel framework directly segments tracts in the field of fibre orientation distribution function peaks without using tractography, image registration or parcellation. This allows to speed up the computational time with respect to traditional methods while providing unprecedented accuracy.

Many extensions of the CNN have been proposed in the literature. In [59], the authors use a $3 \mathrm{D}-\mathrm{CNN}$ that processes multi-modal preoperative brain images and learns a set of supervised features processed by a SVM to predict survival time in patients with high-grade glioma. Another interesting framework based on CNN is the U-net [70], which is developed mainly for biomedical image segmentation and is able to work with few training images or to provide better accuracy. In [60] the U-net is used to segment the kidney and improve the registration accuracy while avoiding artefacts during the free-breathing multi-b-value DW-MRI acquisition.

Similarly to the previous work based on RF, Tanno et al. in [61] show that IQT is a highly ill-posed problem, and training a standard CNN could create inevitable ambiguity in the learning procedure. For this reason, they propose to model uncertainty through a per-patch heteroscedastic noise and Bayesian inferences that extend the standard CNN concept. They show that this leads to obtaining convincing super-resolved DW-MRI and produces tangible benefits in downstream tractography.

Finally, a particular formulation of the CNN developed to control memory usage during network training was proposed in [62]. This framework, evaluated in the paradigm of IQT, is able to improve the prediction results by exploiting a deeper network which, however, requires only a slight memory increase.

\subsection{Deep Autoencoder}

Most of the approaches described so far use a supervised learning strategy to train the networks. This means that each sample has a label used as ground truth during the training. However, in some cases, labels are missing or only partially available and the models need to be trained in an unsupervised fashion. An autoencoder is a special ANN designed exactly for this purpose. In this network, the number of input and output nodes are the same, and the training procedure doesn't require any labels. Instead, it is based on recreating the input data rather than assigning a class label to it. This procedure enables to encode the data in a lower dimensional space and extract the most discriminative features. With the recent advances in deep learning, many autoencoders can be stacked together to create a deep autoencoder architecture. 
An example of this framework was proposed in [64] for a spatio-temporal denoising of contrast-enhanced MRI sequences, nevertheless, it could be applied to DW-MRI data as well. Here, each autoencoder on the stack is trained on a specific subset of the input space to accommodate different noise characteristics and curve prototypes. Spatial dependencies of the pharmacokinetic dynamics are captured by incorporating the curves of neighbouring voxels in the entire process. A stack autoencoder was also used in $[65,66]$ which propose a new noninvasive approach for early classification of renal rejection types after transplant. The developed framework mainly consists of three steps: 1) data co-alignment using a 3D B-spline-based approach and segmentation of kidney tissue with an evolving geometric deformable model guided by a voxel-wise stochastic speed function, 2) construction of a cumulative empirical distribution of ADC at low and high b-values of the segmented kidney taking into account blood perfusion and water diffusion, and 3) classification of acute renal transplant rejection types based on a deep autoencoder with non-negative constraints.

A computer-aided classification method of prostate cancer grade groups using a stacked sparse autoencoder for mpMRI was also proposed in [63]. This special deep framework based on the extraction of high-level features from hand-crafted texture features achieved the first place in a related challenge, winning over 43 methods submitted by 21 groups.

In [67] instead, a Variational Autoencoder (VAE), that is a particular formulation of the standard autoencoder where strong assumptions are made on the distribution of latent variables, was proposed to detect diffusion-space abnormalities in DW-MRI scans of MS patients. Here, only samples from the normal class are available during the training, while test samples are classified as normal or abnormal through the assignment of a novelty score efficiently computed by VAE.

\subsection{Generative Adversarial Network}

A Generative Adversarial Network (GAN) is a deep framework that is particularly useful when the training set is only partially labelled. GAN is a class of ANN which train two separate models that challenge each other in a zero-sum game: the first model is a generative model $G$, and the second is a discriminative model $D$. The first model is trained to learn how to generate data that looks like the real available data, and the second is trained to discriminate the sample generated by $G$ between the real one. Since the aim of $G$ is to understand how to fool the $D$ network this leads to a better generation of realistic samples.

In medical imaging, GAN has shown its success in generating synthetic underrepresented data or for the super-resolution task applied to different image modalities, such as for endomicroscopy images [71], or DW-MRI [68]. Specifically, in this last study, GAN was used to obtain high-resolution images without requiring larger magnetic fields or long scan times. Apart from these examples, GAN has been also used for generating realistic segmentation such as in [69] where an adversarial network which discriminates between expert and generated annotations is used to detect aggressive prostate cancer. This framework, which 
combines an adversarial training with the U-Net model, obtains good results on segmenting the prostates regions along with the targeted cancer nodules.

\section{Discussion and Future Perspectives}

In this review, we have described the current trends and main methods used in machine learning and deep learning for DW-MRI. We have shown that many machine learning methods have already been successfully applied to solve a large variety of problems in DW-MRI, for instance designing better acquisitions and reconstruction tools, mapping microstructure parameters, classifying biomarkers or predicting disease outcomes. We have organized this paper by grouping the methods according to the main methodological classes, explaining their applications and stating where and why that class can be particularly useful. Specifically, in the last few years, a sub-field of machine learning called deep learning has shown a large interest in research and has been employed in many applications, including in the field of DW-MRI. The most likely reason for its success in this area is that DW-MRI has high dimensionality and/or is often coupled with other MRI contrasts, leading to a very large amount of data to be processed, that standard machine learning methods are not able to handle. Moreover, handcrafted features can be difficult to extract in these scenarios since they may be suitable for only one single modality but not adequate for the others. Deep learning -a technique based on the ANN principles- seems particularly suitable to process a large amount of data allowing the model itself to automatically extract the best features. This characteristic seems particularly important to improve the final results of a model.

In conclusion, in the most recent methods described in this review, we report the use of deep learning, not as a single black box, but rather integrated with mathematical modelling to improve the training procedure. For this reason, we believe that in the near future we will see more hybrid models where characteristics of deep learning and traditional modelling are combined together for better analysis and results.

\section{Acknowledgments}

DR and DCA are supported by a project that has received funding from the European Unions Horizon 2020 research and innovation programme under grant agreement number 666992. EPSRC grants EP/M020533/1 and EP/N018702/1 support AI and DCAs work on this topic. UCL EPSRC Centre for Doctor Training in Medical Imaging (EP/L016478/1) funds NG.

\section{References}

1. E. O. Stejskal and T. E. Tanner, "Spin diffusion measurements: spin echoes in the presence of a time-dependent field gradient," Journal of Chemical Physics, vol. 42, pp. 288-292, 1965. 
2. H. Johansen-Berg and T. E. Behrens, Diffusion MRI: from quantitative measurement to in vivo neuroanatomy. Academic Press, 2013.

3. A. R. Padhani, G. Liu, D. Mu-Koh, T. L. Chenevert, H. C. Thoeny, T. Takahara, A. Dzik-Jurasz, B. D. Ross, M. Van Cauteren, D. Collins, D. A. Hammoud, G. J. S. Rustin, B. Taouli, and P. L. Choyke, "Diffusion-weighted magnetic resonance imaging as a cancer biomarker: Consensus and recommendations," Neoplasia, vol. 11, p. $102125,2009$.

4. P. W. Schaefer, P. E. Grant, and R. G. Gonzalez, "Diffusion-weighted mr imaging of the brain," Radiology, vol. 217, pp. 331-345, 2000.

5. P. J. Basser, J. Matiello, and D. Le Bihan, "MR diffusion tensor spectroscopy and imaging," Biophysical Journal, vol. 66, pp. 259-267, 1994.

6. A. L. Alexander, J. E. Lee, M. Lazar, and A. S. Field, "Diffusion tensor imaging of the brain," Neurotherapeutics, vol. 4, p. 316329, 2007.

7. C. A. M. Wheeler-Kingshott, S. J. Hickman, G. J. M. Parker, O. Ciccarelli, M. R. Symms, D. H. Miller, and G. J. Barker, "Investigating cervical spinal cord structure using axial diffusion tensor imaging," NeuroImage, vol. 16, p. 93102, 2002.

8. P. Mukherjee, J. I. Berman, S. W. Chung, C. P. Hess, and R. G. Henry, "Investigating cervical spinal cord structure using axial diffusion tensor imaging," American Journal of Neuroradiology, vol. 29, pp. 632-641, 2008.

9. S. Yoshida, K. Oishi, A. V. Faria, and S. Mori, "Diffusion tensor imaging of normal brain development," Pediatr Radiol, vol. 43, p. 1527, 2013.

10. M. de Groot, L. G. M. Cremers, M. A. Ikram, A. Hofman, G. P. Krestin, A. van der Lugt, W. J. Niessen, and M. W. Vernooij, "White matter degeneration with aging: Longitudinal diffusion mr imaging analysis," Radiology, vol. 279, p. 532541, 2015.

11. A. Lerner, M. A. Mogensen, P. E. Kim, M. S. Shiroishi, D. H. Hwang, and M. Law, "Clinical applications of diffusion tensor imaging," World Neurosurgery, vol. 82, pp. 96-109, 2014.

12. F. Grussu and C. A. M. Gandini Wheeler-Kingshott, The Diffusion of Water (DTI). CRC Press, 2018.

13. D. S. Tuch, T. G. Reese, M. R. Wiegell, and V. J. Wedeen, "Diffusion mri of complex neural architecture," Neurotechnique, vol. 40, pp. 885-895, 2003.

14. D. C. Alexander, T. B. Dyrby, M. Nilsson, and H. Zhang, "Imaging brain microstructure with diffusion mri: practicality and applications," NMR Biomed, vol. e3841, 2017.

15. D. S. Novikov, E. Fieremans, S. N. Jespersen, and V. G. Kiselev, "Quantifying brain microstructure with diffusion mri: Theory and parameter estimation," arXiv, vol. 1612.02059, 2016.

16. A. Ghosh, A. Ianus, and D. C. Alexander, Advanced Diffusion Models. CRC Press, 2018.

17. D. C. Alexander, "A general framework for experiment design in diffusion mri and its application in measuring direct tissue-microstructure features," Magnetic Resonance in Medicine: An Official Journal of the International Society for Magnetic Resonance in Medicine, vol. 60, no. 2, pp. 439-448, 2008.

18. Z. Yang, G. Chen, D. Shen, and P.-T. Yap, "Robust fusion of diffusion mri data for template construction," Scientific reports, vol. 7, no. 1, p. 12950, 2017.

19. M. Reisert, E. Kellner, B. Dhital, J. Hennig, and V. G. Kiselev, "Disentangling micro from mesostructure by diffusion mri: A bayesian approach," Neuroimage, vol. 147, pp. 964-975, 2017.

20. G. L. Nedjati-Gilani, T. Schneider, M. G. Hall, N. Cawley, I. Hill, O. Ciccarelli, I. Drobnjak, C. A. G. Wheeler-Kingshott, and D. C. Alexander, "Machine learn- 
ing based compartment models with permeability for white matter microstructure imaging," NeuroImage, vol. 150, pp. 119-135, 2017.

21. D. C. Alexander, D. Zikic, J. Zhang, H. Zhang, and A. Criminisi, "Image quality transfer via random forest regression: applications in diffusion mri," in International Conference on Medical Image Computing and Computer-Assisted Intervention, pp. 225-232, Springer, 2014.

22. M. Descoteaux, R. Deriche, T. R. Knosche, and A. Anwander, "Deterministic and probabilistic tractography based on complex fibre orientation distributions," IEEE transactions on medical imaging, vol. 28, no. 2, pp. 269-286, 2009.

23. T. Ganepola, Z. Nagy, D. Alexander, and M. Sereno, "An unsupervised group average cortical parcellation using hardi data," in An unsupervised group average cortical parcellation using HARDI data, vol. 2015, p. 221, Organization for Human Brain Mapping, 2015.

24. Y. Guo, Y.-Q. Cai, Z.-L. Cai, Y.-G. Gao, N.-Y. An, L. Ma, S. Mahankali, and J.-H. Gao, "Differentiation of clinically benign and malignant breast lesions using diffusion-weighted imaging," Journal of magnetic resonance imaging, vol. 16, no. 2, pp. $172-178,2002$.

25. H. C. Thoeny and B. D. Ross, "Predicting and monitoring cancer treatment response with diffusion-weighted mri," Journal of Magnetic Resonance Imaging, vol. 32, no. 1, pp. 2-16, 2010.

26. D. A. Yablonskiy and A. L. Sukstanskii, "Theoretical models of the diffusion weighted mr signal," NMR in Biomedicine, vol. 23, no. 7, pp. 661-681, 2010.

27. C. Lenglet, J. S. Campbell, M. Descoteaux, G. Haro, P. Savadjiev, D. Wassermann, A. Anwander, R. Deriche, G. B. Pike, G. Sapiro, et al., "Mathematical methods for diffusion mri processing," Neuroimage, vol. 45, no. 1, pp. S111-S122, 2009.

28. D. Ravı, C. Wong, F. Deligianni, M. Berthelot, J. Andreu-Perez, B. Lo, and G.Z. Yang, "Deep learning for health informatics," IEEE journal of biomedical and health informatics, vol. 21, no. 1, pp. 4-21, 2017.

29. J. M. Provenzale, L. Liang, D. DeLong, and L. E. White, "Diffusion tensor imaging assessment of brain white matter maturation during the first postnatal year," American Journal of Roentgenology, vol. 189, no. 2, pp. 476-486, 2007.

30. A. Dzik-Jurasz, C. Domenig, M. George, J. Wolber, A. Padhani, G. Brown, and S. Doran, "Diffusion mri for prediction of response of rectal cancer to chemoradiation," The Lancet, vol. 360, no. 9329, pp. 307-308, 2002.

31. T. Billiet, M. Vandenbulcke, B. Mädler, R. Peeters, T. Dhollander, H. Zhang, S. Deprez, B. R. Van den Bergh, S. Sunaert, and L. Emsell, "Age-related microstructural differences quantified using myelin water imaging and advanced diffusion mri," Neurobiology of aging, vol. 36, no. 6, pp. 2107-2121, 2015.

32. G. L. Nedjati-Gilani, T. Schneider, M. G. Hall, C. A. Wheeler-Kingshott, and D. C. Alexander, "Machine learning based compartment models with permeability for white matter microstructure imaging," in International Conference on Medical Image Computing and Computer-Assisted Intervention, pp. 257-264, Springer, 2014.

33. R. Fick, N. Sepasian, M. Pizzolato, A. Ianus, and R. Deriche, "Assessing the feasibility of estimating axon diameter using diffusion models and machine learning," in IEEE International Symposium on Biomedical Imaging (ISBI), 2017.

34. S. Mesaros, M. Rocca, K. Kacar, J. Kostic, M. Copetti, T. Stosic-Opincal, P. Preziosa, S. Sala, G. Riccitelli, M. Horsfield, et al., "Diffusion tensor mri tractography and cognitive impairment in multiple sclerosis," Neurology, pp. WNL0b013e31824d5859, 2012. 
35. S. R. McWhinney, A. Tremblay, T. M. Chevalier, V. K. Lim, and A. J. Newman, "Using cforest to analyze diffusion tensor imaging data: a study of white matter integrity in healthy aging," Brain connectivity, vol. 6, no. 10, pp. 747-758, 2016.

36. P. F. Neher, M.-A. Cote, J.-C. Houde, M. Descoteaux, and K. H. Maier-Hein, "Fiber tractography using machine learning," Neuroimage, vol. 158, pp. 417-429, 2017.

37. J. Mitra, P. Bourgeat, J. Fripp, S. Ghose, S. Rose, O. Salvado, A. Connelly, B. Campbell, S. Palmer, G. Sharma, et al., "Lesion segmentation from multimodal mri using random forest following ischemic stroke," NeuroImage, vol. 98, pp. 324-335, 2014.

38. D. C. Alexander, D. Zikic, A. Ghosh, R. Tanno, V. Wottschel, J. Zhang, E. Kaden, T. B. Dyrby, S. N. Sotiropoulos, H. Zhang, et al., "Image quality transfer and applications in diffusion mri," Neuroimage, vol. 152, pp. 283-298, 2017.

39. R. Tanno, A. Ghosh, F. Grussu, E. Kaden, A. Criminisi, and D. C. Alexander, "Bayesian image quality transfer," in International Conference on Medical Image Computing and Computer-Assisted Intervention, pp. 265-273, Springer, 2016.

40. A. E. Raftery, "Approximate bayes factors and accounting for model uncertainty in generalised linear models," Biometrika, vol. 83, no. 2, pp. 251-266, 1996.

41. I. Park, H. K. Amarchinta, and R. V. Grandhi, "A bayesian approach for quantification of model uncertainty," Reliability Engineering 85 System Safety, vol. 95, no. 7, pp. 777-785, 2010.

42. O. Friman, G. Farneback, and C.-F. Westin, "A bayesian approach for stochastic white matter tractography," IEEE transactions on medical imaging, vol. 25, no. 8, pp. 965-978, 2006.

43. K. E. Emblem, P. Due-Tonnessen, J. K. Hald, A. Bjornerud, M. C. Pinho, D. Scheie, L. R. Schad, T. R. Meling, and F. G. Zoellner, "Machine learning in preoperative glioma mri: Survival associations by perfusion-based support vector machine outperforms traditional mri," Journal of magnetic resonance imaging, vol. 40, no. 1, pp. 47-54, 2014.

44. S. Schnell, D. Saur, B. Kreher, J. Hennig, H. Burkhardt, and V. G. Kiselev, "Fully automated classification of hardi in vivo data using a support vector machine," NeuroImage, vol. 46, no. 3, pp. 642-651, 2009.

45. D.-L. Feis, K. H. Brodersen, D. Y. von Cramon, E. Luders, and M. Tittgemeyer, "Decoding gender dimorphism of the human brain using multimodal anatomical and diffusion mri data," Neuroimage, vol. 70, pp. 250-257, 2013.

46. Y. Artan, M. A. Haider, D. L. Langer, T. H. van der Kwast, A. J. Evans, Y. Yang, M. N. Wernick, J. Trachtenberg, and I. S. Yetik, "Prostate cancer localization with multispectral mri using cost-sensitive support vector machines and conditional random fields," IEEE Transactions on Image Processing, vol. 19, no. 9, pp. 2444$2455,2010$.

47. S. Ozer, M. A. Haider, D. L. Langer, T. H. van der Kwast, A. J. Evans, M. N. Wernick, J. Trachtenberg, and I. S. Yetik, "Prostate cancer localization with multispectral mri based on relevance vector machines," in Biomedical Imaging: From Nano to Macro, 2009. ISBI'09. IEEE International Symposium on, pp. 73-76, IEEE, 2009.

48. H. Bagher-Ebadian, K. Jafari-Khouzani, P. D. Mitsias, M. Lu, H. Soltanian-Zadeh, M. Chopp, and J. R. Ewing, "Predicting final extent of ischemic infarction using artificial neural network analysis of multi-parametric mri in patients with stroke," PloS one, vol. 6, no. 8, p. e22626, 2011. 
49. M. Bertleff, S. Domsch, S. Weingärtner, J. Zapp, K. O'Brien, M. Barth, and L. R. Schad, "Diffusion parameter mapping with the combined intravoxel incoherent motion and kurtosis model using artificial neural networks at 3 t," NMR in Biomedicine, vol. 30, no. 12, p. e3833, 2017.

50. S. Koppers, C. Haarburger, and D. Merhof, "Diffusion mri signal augmentation: from single shell to multi shell with deep learning," in International Conference on Medical Image Computing and Computer-Assisted Intervention, pp. 61-70, Springer, 2016.

51. V. Golkov, A. Dosovitskiy, P. Sämann, J. I. Sperl, T. Sprenger, M. Czisch, M. I. Menzel, P. A. Gómez, A. Haase, T. Brox, et al., "q-space deep learning for twelve-fold shorter and model-free diffusion mri scans," in International Conference on Medical Image Computing and Computer-Assisted Intervention, pp. 37-44, Springer, 2015.

52. I. D. Hill, M. Palombo, M. D. Santin, F. Branzoli, A.-C. Philippe, D. Wassermann, M.-S. Aigrot, B. Stankoff, H. Zhang, S. Lehericy, et al., "Deep neural network based framework for in-vivo axonal permeability estimation,"

53. H. K. van der Burgh, R. Schmidt, H.-J. Westeneng, M. A. de Reus, L. H. van den Berg, and M. P. van den Heuvel, "Deep learning predictions of survival based on mri in amyotrophic lateral sclerosis," NeuroImage: Clinical, vol. 13, pp. 361-369, 2017.

54. T. A. Roberts, B. Hipwell, G. Agliardi, A. d'Esposito, V. Taylor, M. F. Lythgoe, and S. Walker-Samuel, "Deep learning diffusion fingerprinting to detect brain tumour response to chemotherapy," bioRxiv, p. 193730, 2017.

55. S. Koppers and D. Merhof, "Direct estimation of fiber orientations using deep learning in diffusion imaging," in International Workshop on Machine Learning in Medical Imaging, pp. 53-60, Springer, 2016.

56. S. Trebeschi, J. J. van Griethuysen, D. M. Lambregts, M. J. Lahaye, C. Parmer, F. C. Bakers, N. H. Peters, R. G. Beets-Tan, and H. J. Aerts, "Deep learning for fully-automated localization and segmentation of rectal cancer on multiparametric mr," Scientific reports, vol. 7, no. 1, p. 5301, 2017.

57. J. Wasserthal, P. Neher, and K. H. Maier-Hein, "Tractseg-fast and accurate white matter tract segmentation," NeuroImage, vol. 183, pp. 239-253, 2018.

58. T. Clark, J. Zhang, S. Baig, A. Wong, M. A. Haider, and F. Khalvati, "Fully automated segmentation of prostate whole gland and transition zone in diffusionweighted mri using convolutional neural networks," Journal of Medical Imaging, vol. 4, no. 4, p. 041307, 2017.

59. D. Nie, H. Zhang, E. Adeli, L. Liu, and D. Shen, "3d deep learning for multimodal imaging-guided survival time prediction of brain tumor patients," in International Conference on Medical Image Computing and Computer-Assisted Intervention, pp. 212-220, Springer, 2016.

60. J. Lv, W. Huang, J. Zhang, and X. Wang, "Performance of u-net based pyramidal lucas-kanade registration on free-breathing multi-b-value diffusion mri of the kidney," The British journal of radiology, vol. 91, no. 1086, p. 20170813, 2018.

61. R. Tanno, D. E. Worrall, A. Ghosh, E. Kaden, S. N. Sotiropoulos, A. Criminisi, and D. C. Alexander, "Bayesian image quality transfer with cnns: Exploring uncertainty in dmri super-resolution," in International Conference on Medical Image Computing and Computer-Assisted Intervention, pp. 611-619, Springer, 2017.

62. S. B. Blumberg, R. Tanno, I. Kokkinos, and D. C. Alexander, "Deeper image quality transfer: Training low-memory neural networks for 3d images," in International Conference on Medical Image Computing and Computer-Assisted Intervention, pp. 118-125, Springer, 2018. 
63. B. Abraham and M. S. Nair, "Computer-aided classification of prostate cancer grade groups from mri images using texture features and stacked sparse autoencoder," Computerized Medical Imaging and Graphics, vol. 69, pp. 60-68, 2018.

64. A. Benou, R. Veksler, A. Friedman, and T. R. Raviv, "Ensemble of expert deep neural networks for spatio-temporal denoising of contrast-enhanced mri sequences," Medical image analysis, vol. 42, pp. 145-159, 2017.

65. M. Shehata, F. Khalifa, E. Hollis, A. Soliman, E. Hosseini-Asl, M. A. El-Ghar, M. El-Baz, A. C. Dwyer, A. El-Baz, and R. Keynton, "A new non-invasive approach for early classification of renal rejection types using diffusion-weighted mri," in Image Processing (ICIP), 2016 IEEE International Conference on, pp. 136-140, IEEE, 2016.

66. M. Shehata, F. Khalifa, A. Soliman, M. Ghazal, F. Taher, M. A. El-Ghar, A. Dwyer, G. Gimel'farb, R. Keynton, and A. El-Baz, "Computer-aided diagnostic system for early detection of acute renal transplant rejection using diffusionweighted mri," IEEE Transactions on Biomedical Engineering, 2018.

67. A. Vasilev, V. Golkov, I. Lipp, E. Sgarlata, V. Tomassini, D. K. Jones, and D. Cremers, "q-space novelty detection with variational autoencoders," arXiv preprint arXiv:1806.02997, 2018.

68. E. Albay, U. Demir, and G. Unal, "Diffusion mri spatial super-resolution using generative adversarial networks," in International Workshop on PRedictive Intelligence In MEdicine, pp. 155-163, Springer, 2018.

69. S. Kohl, D. Bonekamp, H.-P. Schlemmer, K. Yaqubi, M. Hohenfellner, B. Hadaschik, J.-P. Radtke, and K. Maier-Hein, "Adversarial networks for the detection of aggressive prostate cancer," arXiv preprint arXiv:1702.08014, 2017.

70. O. Ronneberger, P. Fischer, and T. Brox, "U-net: Convolutional networks for biomedical image segmentation," in International Conference on Medical image computing and computer-assisted intervention, pp. 234-241, Springer, 2015.

71. D. Ravì, A. B. Szczotka, D. I. Shakir, S. P. Pereira, and T. Vercauteren, "Adversarial training with cycle consistency for unsupervised super-resolution in endomicroscopy," 2018. 